\title{
The effects of visual search efficiency on object-based attention
}

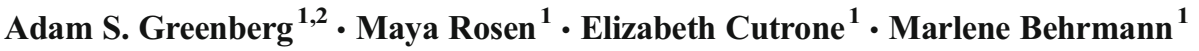

Published online: 2 April 2015

(C) The Psychonomic Society, Inc. 2015

\begin{abstract}
The attentional prioritization hypothesis of objectbased attention (Shomstein \& Yantis in Perception \& Psychophysics, 64, 41-51, 2002) suggests a two-stage selection process comprising an automatic spatial gradient and flexible strategic (prioritization) selection. The combined attentional priorities of these two stages of object-based selection determine the order in which participants will search the display for the presence of a target. The strategic process has often been likened to a prioritized visual search. By modifying the double-rectangle cueing paradigm (Egly, Driver, \& Rafal in Journal of Experimental Psychology: General, 123, 161-177, 1994) and placing it in the context of a larger-scale visual search, we examined how the prioritization search is affected by search efficiency. By probing both targets located on the cued object and targets external to the cued object, we found that the attentional priority surrounding a selected object is strongly modulated by search mode. However, the ordering of the prioritization search is unaffected by search mode. The data also provide evidence that standard spatial visual search and object-based prioritization search may rely on distinct mechanisms. These results provide insight into the interactions between the mode of visual search and object-based
\end{abstract}

Electronic supplementary material The online version of this article (doi:10.3758/s13414-015-0892-7) contains supplementary material, which is available to authorized users.

Adam S. Greenberg

agreenb@uwm.edu

1 Department of Psychology and Center for the Neural Basis of Cognition, Carnegie Mellon University, Pittsburgh, PA, USA

2 Department of Psychology, University of Wisconsin-Milwaukee, P.O. Box 413, Milwaukee, WI 53201, USA selection, and help define the modulatory consequences of search efficiency for object-based attention.

Keywords Spatial cueing $\cdot$ Object representation $\cdot$ Search Mode $\cdot$ Exogenous cue

Visual attention operates selectively to ensure that cognitive processes are restricted to the most critical aspects of the input as they are propagated through the visual system. It has been widely shown that attentional mechanisms can accomplish this information selection in various ways - by selecting on the basis of spatial locations, features (color values, motion directions, etc.), and objectness. Object-based attentional selection has largely been studied through variations of a procedure introduced by Egly, Driver, and Rafal (1994), a cueing paradigm in which the target appears at the cued location, at an invalid location on the same object, or at an invalid location external to the cued object, with both invalid locations being equidistant from the cue. Under such conditions, participants not only show a validity effect (best report of target when it appears at the cued location), but also identify targets on the currently attended object better than those appearing elsewhere. The former result reflects the facilitation afforded by space-based attention, and the latter provides the key demonstration of the object-based attention (OBA) advantage (Egly et al., 1994).

The most robust account of OBA, to date, is the attentional prioritization hypothesis proposed by Shomstein and Yantis (2002). This theory posits the following two stages of selection: (1) an automatic spatial selection, in the form of a gradient centered on the cued location, and (2) a strategic stage, by which locations of perceived higher target probability are 
prioritized above locations of perceived lower target probability. The combined attentional priorities of these two stages of object-based selection determine the order in which participants will search the display for the presence of a target. This has often been likened to a visual search, during which the search order is determined by the combined priorities of items along many different feature dimensions (Wolfe, 1994). However, the difference during OBA is that perceptual object organization further constrains attentional prioritization (Shomstein, 2012).

The speed and nature of information transmission through the visual system has been assayed primarily by measuring performance using a variety of visual search paradigms (Egeth, Jonides, \& Wall, 1972; Treisman, 1982). In these paradigms, the observer typically searches for a predefined target that is embedded among a host of lures or distracter items. The mode of information transmission refers to the extent to which the target search is conducted efficiently across all elements of the display, versus inefficiently, so that each item must be examined in sequence. ${ }^{1}$ In such studies, the primary manipulation is typically the relationship between the features of the target and distracters, along with varying the number of distracters in the display (Wolfe \& Horowitz, 2004). For example, certain relationships (e.g., a green letter $\mathrm{L}$ among all white Ts and Ls) result in fast RTs that change very little as additional distracters are added to the display. These results are referred to as "pop-out" and typify an efficient visual search. Other target-distracter relationships (e.g., a green L among purple, blue, and yellow Ts and Ls) are more difficult, require an inefficient search, and result in RTs that increase linearly as additional distracters are added. There is wide agreement that these search slopes are hallmarks of efficient (flat slope) and inefficient (linearly increasing slope) visual search, respectively (Treisman \& Gelade, 1980).

In this study, we were interested in probing the representational basis of attentional selection under varying conditions of search efficiency. The critical question concerned the computations activated when participants are searching for a target embedded among multiple distracters while a perceptually organized object is present in the visual field. As such, we have wedded the standard visual search and cued-rectangle paradigms to explore the interaction between search efficiency and object-based attentional selection. Indeed, our conjoined

\footnotetext{
${ }^{1}$ We favor the use of efficient and inefficient search over the historical terms parallel and serial, respectively, due to the controversy involving the mechanisms inferred from search slopes (Wolfe, 1998). Efficiency is, obviously, a matter of degree, not an absolute property. Throughout this article, our reference to efficient versus inefficient processing indicates a relative manipulation. We only wish to convey that our participants were in two different modes of processing in the conditions/data we label efficient and inefficient. The continuum between efficient and inefficient was not explicitly manipulated in this study; that is, participants may have been conducting a more purely efficient search and a less purely inefficient search (or vice versa).
}

displays may be a closer approximation to the processes that occur naturalistically when an observer searches for an item in an everyday scene.

By combining the visual search and cued-rectangle paradigms, we were able to ask two important questions about the attentional prioritization hypothesis of OBA that have previously not been addressed in the literature: (1) Is the prioritization search affected by mode of visual search?, and (2) Does the prioritization search share a common search mechanism with standard visual search processes?

The visual displays employed here contained two general classes of locations where targets could appear on each trial: target locations on the cued object, and target locations external to the cued object (see Fig. 1A). By varying the locations of external targets "nearby" (in close proximity to) the object, we could characterize the effect of search efficiency on the two selection stages proposed by the prioritization account of OBA: the automatic spatial gradient and the flexible strategic selection.

Previous work had provided some insight into the interactions between search efficiency and object-based selection.

A

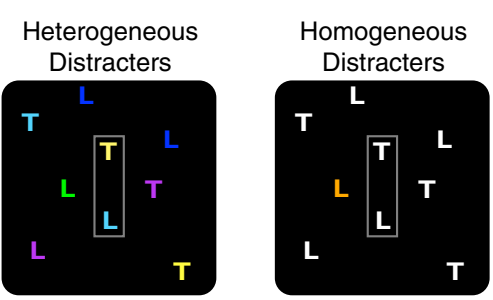

B
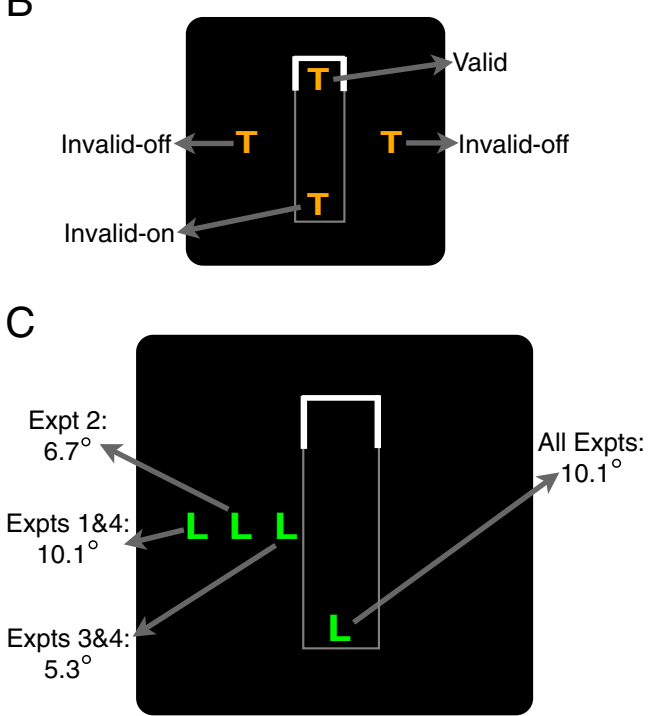

Fig. 1 Example stimulus displays for Experiments 1, 2, and 3. (A) Examples of the target/distracter display. The left image shows a green target with eight heterogeneous distracter colors; the right image shows an orange target with eight homogeneous distracter colors. (B) Locations of the invalid-on target (same for all experiments) and invalid-off targets (different for Exps. 1, 2, and 3). (C) The distances from the center of the exogenous cue are also noted 
For example, using overlapped stimuli (rather than cued objects; cf. Duncan, 1984) in a conjunction search, Goldsmith (1998) found evidence suggesting that the OBA advantage is primarily present during inefficient visual search and largely absent during efficient search. Relatedly, Rensink and Enns (1995) used Müller-Lyer stimuli to show that rapid (efficient) visual search processes do not have access to grouped object structure. Importantly, we are unaware of any studies that have systematically probed locations nearby a cued object to measure the effects of the attentional gradient in the context of efficient/inefficient visual search.

Recent work has nicely characterized the gradient of attention within a selected object during object-based selection (Hollingworth, Maxcey-Richard, \& Vecera, 2012). The authors found that the object-based attentional gradient was maximal at the cued location and diminished with distance from the cue. Although the authors supported a different mechanism for the allocation of attentional priority during object-based selection, in which object structure constrains the spread of attention (the grouped-array account; Vecera \& Farah, 1994), they also left open the notion that attention may not be strictly bounded by the selected object. That is, "object boundaries may retard the spread of attention but do not necessarily eliminate it" (Hollingworth et al., 2012). Nevertheless, there has been one attempt to characterize the spatial gradient outside a cued object during OBA. In this work, the authors showed that nearby locations inherit some of the facilitation afforded to the cued object, the effects of which fall off linearly as a function of the target distance to the object (Kravitz \& Behrmann, 2008). The present study is largely concerned with the fates of targets in these nearby locations during a larger-scale visual search. Importantly, Kravitz and Behrmann manipulated the target distance to the object's center of mass, not the distance to the spatial cue (all targets were equidistant to the cue). Therefore, they were unable to explore the effects of the spatial gradient as a function of the spacebased selection component of their data. Here, we manipulated both the distance to the object's center of mass and, simultaneously, the distance to the spatial cue. Combined with our manipulation of search efficiency, this allowed us to ask the following questions: Would the linear gradient shown by Kravitz and Behrmann hold during both efficient and inefficient visual search? How would the prioritization mechanism be affected by visual search mode? And, finally, can we distinguish between spatial visual search and prioritized objectbased visual search mechanisms?

\section{Experiment 1}

In this first experiment, we measured the effects of space- and object-based attentional selection while participants concurrently searched for a target embedded in multiple distracters.
As such, we merged a standard visual search paradigm with a variant of the classic cued-rectangle paradigm developed by Egly and colleagues (1994; see Fig. 1). The critical comparisons lay in the RT difference between (a) targets that appeared in cued versus noncued locations of the object (space-based attention) and (b) targets that appeared in the noncued location on the object (invalid-on condition) versus targets that appeared to the left or right of the object (invalid-off condition). Since, in case (b), the target locations were equated for distance to the exogenous cue (and target probability), any acceleration of RTs in the invalid-on condition would likely be due to prior attentional selection of the rectangle on which it was located. Furthermore, we examined the space- and objectbased effects [cases (a) and (b) above, respectively], as well as the detection of the invalid-off target as a function of search mode (efficient vs. inefficient), and thereby characterized the changes in object selection affordances as information transmission through the visual system was varied.

\section{Method}

Participants A group of 32 participants (mean age $=32.0 ; 18$ male, 14 female) from the Pittsburgh university community provided written informed consent. Participants were recruited through an online research volunteer participant pool administered by the Center for Behavioral and Decision Research at Carnegie Mellon University (CMU). Here and in all subsequent experiments, participants were paid the standard hourly rate as approved by the CMU Institutional Review Board. All volunteers had normal or corrected-to-normal vision.

Apparatus and stimuli The stimuli were presented on a 17in. CRT monitor at a resolution of $1,024 \times 768$ pixels driven by a Dell computer running Windows XP and programmed in the MATLAB software platform with Psychophysics Toolbox extensions (Brainard, 1997; Pelli, 1997). Participants viewed the monitor in a soundproof, dimly lit room at a distance of approximately $57 \mathrm{~cm}$, ensured by the use of a chinrest.

As is shown in Fig. 1, a single vertical rectangle $\left(1.7^{\circ}\right.$ wide, $11.4^{\circ}$ long) was displayed at the center of the screen. The rectangle's outline $\left(0.1^{\circ}\right.$ thick $)$ was rendered in median gray on a black background. The exogenous cue was a brightening of one end (three sides) of the rectangle, achieved by displaying three white lines (each $0.3^{\circ}$ thick, $1.7^{\circ}$ long) in place of the end portion of the rectangle's existing gray lines. Target letters were rendered in a fixed-width font, subtending $0.5^{\circ}$ in length and width. The targets were colored either green (RGB: [0 255 0]) or orange [139 90 0], a between-subjects manipulation. Two letters appeared within the outline of the rectangle on every trial, one at each end. These letters were each centered left-to-right within the rectangle and positioned so that their centers were $0.65^{\circ}$ from the near end (top or 
bottom) of the rectangle. The two letters were $10.1^{\circ}$ apart (center-to-center). A letter also appeared at one of the two invalid-off object locations (chosen randomly) on each trial. And, depending on the number of distracters on a given trial, letters could also appear at one (or more) of 13 possible pseudorandom locations elsewhere on the screen. Therefore, letters could appear at a total of 17 locations on the screen, and five, nine, or 13 letters appeared on each trial, one of which was the target. Distracters were either all colored white (homogeneous distracter condition) or, in the heterogeneous distracter condition, equal numbers of cyan [0 255255 ], yellow [255 255 0], blue [0 0 255], and purple [200 0 200] distracters. Example target/distracter displays are shown in Fig. 1A.

Design The exogenous cue appeared equally likely at either the top or bottom of the rectangle (randomly) on each trial. The following four trial types were defined by the target location (see Fig. 1B): valid trials $(64.8 \%$; target at the cued location, on the rectangle), invalid-on (12.4\%; target at the far, noncued end of the rectangle), invalid-off (12.4\%; target to either the left or right of the rectangle), and background (10.4\%; target at a pseudorandom location, a far distance from the cue). The distances between the cue and target were equal $\left(10.1^{\circ}\right)$ in the invalid-on and invalid-off conditions. One, and only one, target was present on each trial (no target-absent trials), whereas the display contained four, eight, or 12 distracters (chosen randomly) on each trial. Additionally, we created two distracter color conditions: homogeneous (all distracters colored white) or heterogeneous (equal numbers of distracters colored cyan, yellow, blue, and purple). Participants performed two 1-h sessions, beginning $24 \mathrm{~h}$ apart. Each session contained trials of either all homogeneous or all heterogeneous distracters (order counterbalanced across participants). Half the participants were (randomly) assigned the target color green, and the other half orange. Each session contained two short (20 trials) practice blocks followed by eight blocks of 160 trials each, for a total of 2,560 experimental trials per participant. All blocks contained trials of each type (with distracter color as a between-subjects variable), according to the contingencies stated above. Participants were forced to take a (minimum) 30-s break between blocks to avoid fatigue.

Procedure Trials began with the centered rectangle displayed alone for $500 \mathrm{~ms}$. The exogenous cue was then displayed for $100 \mathrm{~ms}$, followed by a $200-\mathrm{ms}$ interstimulus interval before the target/distracter letters appeared. Thus, the cue-target stimulus onset asynchrony was $300 \mathrm{~ms}$. Letters were presented for a maximum of 2,000 ms, during which participants were instructed to respond "as quickly and accurately as possible" by pressing the key (on a standard computer keyboard) corresponding to the identity ("T" or " $\mathrm{L}$ ") of the target. The target was defined as the only green (or orange, for half of the participants) letter in the display. Response times (RTs) were measured with a precision of less than $1 \mathrm{~ms}$. After response (or $2,000 \mathrm{~ms}$ ), the screen turned black for a variable intertrial interval (ITI) of 400,600 , or $800 \mathrm{~ms}$ before the next trial began. If participants responded incorrectly (or failed to respond), they heard a low-frequency beep (150-ms duration) during the subsequent ITI, as negative feedback.

\section{Results and discussion}

The data, displayed in Fig. 2, showed increases in RTs between target location conditions, in the following order: valid $<$ invalid-on $<$ invalid-off $<$ background. This held for both homogeneous and heterogeneous distracters. Number of distracters did not increase RTs in the homogeneous condition, but it did in the heterogeneous condition, though only for the background target location trials.

Anticipatory responses (less than 150 -ms RTs; $M=1.8$ trials/participant, $S D=3.9$ ) and incorrect trials were excluded from the analyses. Accuracy was at or near ceiling in all
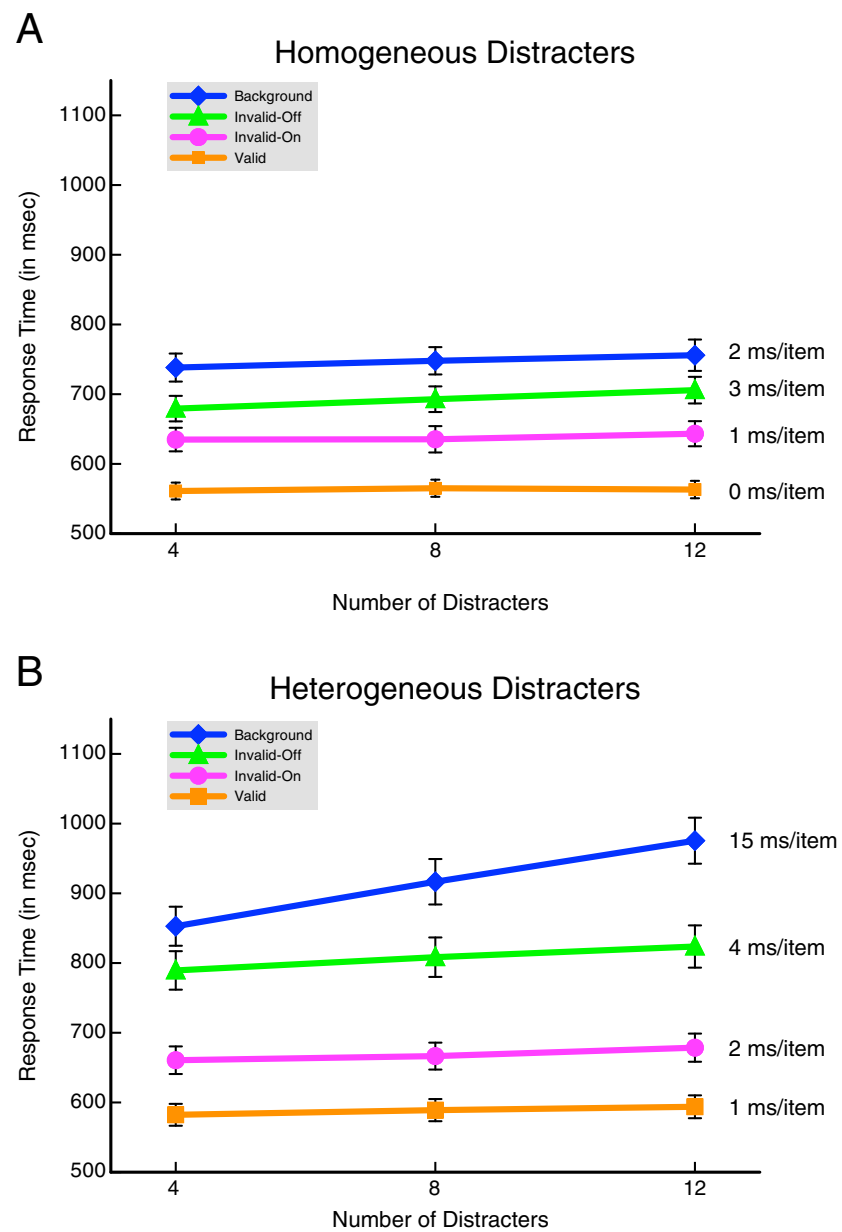

Fig. 2 Response time data for Experiment 1. The four target location condition RTs are plotted against the number of distracters for the homogeneous (A) and heterogeneous (B) distracter conditions. To the right of each line, the search slopes are listed in milliseconds/item 
conditions (overall accuracy $M=.958, S D=.038$ ). An independent-samples $t$ test indicated no accuracy difference between the participants assigned green $(M=.963, S D=$ $.032)$ versus orange $(M=.953, S D=.044)$ targets, $t(30)=$ $0.745, p=.462, d=0.27$. Accuracy was significantly higher on homogeneous-distracter trials $(M=.966, S D=.034)$ than on heterogeneous-distracter trials $(M=.950, S D=.049)$, paired $t(31)=2.37, p=.02, d=0.37$. Other planned tests on accuracy were not significant.

An omnibus analysis of variance (ANOVA) on RTs yielded no significant main effect or interactions with the betweensubjects factor Target Color. Therefore, all further analyses were collapsed across this manipulation. A within-subjects three-way ANOVA (see Fig. 2) with the factors Distracter Colors (homogeneous, heterogeneous), Target Location (valid, invalid-on, invalid-off, background), and Number of Distracters (four, eight, 12) revealed significant main effects of distracter colors $[F(1,31)=48.42, M S E=28,340.7, p<$ $\left..001, \eta_{\mathrm{p}}{ }^{2}=.61\right]$, target location $[F(3,93)=173.77, M S E=13$, $\left.735.6, p<.001, \eta_{\mathrm{p}}{ }^{2}=.85\right]$, and number of distracters $[F(2,62)$ $\left.=75.33, M S E=772.9, p<.001, \eta_{\mathrm{p}}{ }^{2}=.71\right]$. This was qualified by significant interactions between distracter colors and target location $\left[F(3,93)=64.88, M S E=3,504.2, p<.001, \eta_{\mathrm{p}}{ }^{2}=\right.$ $.68]$, distracter colors and number of distracters $[F(2,62)=$ 16.10, MSE $\left.=1076.0, p<.001, \eta_{\mathrm{p}}{ }^{2}=.34\right]$, target location and number of distracters $[F(6,186)=22.17, M S E=592.3, p<$ $\left..001, \eta_{\mathrm{p}}{ }^{2}=.42\right]$, and a significant three-way interaction $[F(6$, $\left.186)=12.86, M S E=717.7, p<.001, \eta_{\mathrm{p}}{ }^{2}=.29\right]$.

In order to determine whether our distracter colors manipulation successfully encouraged participants to conduct an inefficient (heterogeneous condition) versus an efficient (homogeneous condition) search, we performed post-hoc analyses using Tukey's HSD. The results indicated that RTs to background targets increased significantly with additional distracters in the heterogeneous (all $p \mathrm{~s}<.01 ; 15.33 \mathrm{~ms} /$ item), but not in the homogeneous (all $p \mathrm{~s}>.05 ; 2.22 \mathrm{~ms} /$ item) condition.

Having established that participants used different search modes in accordance with our distracter color manipulation, we now examine the three key target location conditions to uncover the attention cueing effects in the two search modes. Like all studies that have made use of Egly et al.'s (1994) cued-rectangle display, we were able to dissociate the effects of space-based selection from those of object-based selection. First, the space-based effect (mean invalid-on RT - mean valid RT) was the same during heterogeneous/inefficient search ( $M$ $=80 \mathrm{~ms}, S D=79)$ and homogeneous/efficient search $(M=$ $75 \mathrm{~ms}, S D=70$ ), suggesting that search mode had no effect on the allocation of attention within the cued object. Post-hoc Tukey tests confirmed that there was no significant difference in either the valid or invalid-on RTs between heterogeneous and homogeneous distracter colors (all $p \mathrm{~s}>.05$ ). Second, with respect to the object-based effects (mean invalid-off RT mean invalid-on RT; equidistant from cue position), Tukey tests revealed a robust object-based advantage for invalid targets appearing on the cued object, as compared with equidistant targets not on the object (all $p$ s $<.01$ ). In addition, unlike in the space-based effects, we found a significant RT difference in the object-based effects between inefficient and efficient search: Post-hoc Tukey tests revealed that invalid-off target RTs were significantly slower with heterogeneous than with homogeneous distracters for all numbers of distracters tested (all $p \mathrm{~s}<.01$ ). The mean object-based effects were approximately $2 \frac{1}{2}$ times larger during inefficient $(M=139 \mathrm{~ms})$ than during efficient ( $M=55 \mathrm{~ms}$; see Table 1) search.

In summary, our manipulation of distracter colors successfully led participants to adopt either an inefficient (heterogeneous condition) or efficient (homogeneous condition) search. This distinction enabled us to examine how the mode of visual search affects attentional selection from spaceand object-based representations. Space-based effects were identical for both visual search modes, suggesting that items located on the cued object are isolated against the effects of search efficiency. With respect to object-based attention, in contrast, participants were slower to respond to nearby offobject targets (invalid-off) during an inefficient (vs. an efficient) search; the OBA advantage afforded targets on the cued object was consequently much larger during inefficient than during efficient search (though it was significantly large in

Table 1 Object-based and space-based response time (RT) cueing effects (in milliseconds) for all experiments

\begin{tabular}{lllll}
\hline & \multicolumn{2}{l}{ Mean Object-Based RT Effects } & & \multicolumn{2}{c}{ Mean Space-Based RT Effects } \\
\cline { 2 - 4 } & Inefficient Search & Efficient Search & Inefficient Search & Efficient Search \\
\hline Exp. 1 & $139(14)^{*}$ & $55(6)^{*}$ & $80(14)^{*}$ & $75(12)^{*}$ \\
Exp. 2 & $75(12)^{*}$ & $17(6)^{*}$ & $76(13)^{*}$ & $74(10)^{*}$ \\
Exp. 3 & $22(18)$ & $-7(9)$ & $95(24)^{*}$ & $74(16)^{*}$ \\
Exp. 4 (near) & $40(14)^{*}$ & $-8(8)$ & $62(12)^{*}$ & $64(10)^{*}$ \\
Exp. 4 (far) & $149(20)^{*}$ & $47(8)^{*}$ & & $60(13)^{*}$ \\
Exp. S1 & $156(18)^{*}$ & $57(10)^{*}$ & & $59(10)^{*}$ \\
\hline
\end{tabular}

Values in parentheses are SEMs. Asterisks indicate significant difference from zero (all $p \mathrm{~s}<.01$ ) 
both conditions) and was driven by the differences in the invalid-off condition (see Fig. 2).

The magnitude of the mean OBA advantage (especially during inefficient search, $139 \mathrm{~ms}$ ) was much larger than those typically reported in the literature. The original effects reported by Egly and colleagues (1994), using a simple detection task, were on the order of $15 \mathrm{~ms}$. A brief review of the recent literature shows that the reported significant object-based effects range from 5 to $42 \mathrm{~ms}$ (Chou \& Yeh, 2012; Drummond \& Shomstein, 2010; Hollingworth et al., 2012; Lin \& Yeh, 2011, 2012; Pilz, Roggeveen, Creighton, Bennett, \& Sekuler, 2012; Yeari \& Goldsmith, 2010), the latter of which is less than $1 / 3$ of what we observed during inefficient search. Of course, this difference could be due to any number of deviations of the present paradigm from the typical doublerectangle display. However, one explanation hinges on performance during the invalid-off condition.

The pattern of observed results suggests that, after the target and distracter letters appear, participants first search for the target at the cued, valid location. Next, they try the invalid-on location, which benefits both from its location on the cued object and its status as the second-highest probable target location. Both the ordering of this search and the performance magnitude did not differ as a function of the visual search mode. In agreement with the predictions of the prioritization hypothesis of OBA, the invalid-off target was the next location searched. However, performance was drastically different during inefficient than during efficient visual search. Because the invalid-off location was outside the bounds of the object, it became more difficult to segment this item from the distracters. During efficient search, this item is more easily attended because of its joint status as both a color singleton and a high-priority item in the search array (due to the matched target probability with the invalid-on location). However, during inefficient search, this location is no longer a color singleton and is, therefore, more confusable with the background items, leading to reduced performance.

In order to probe the spatial gradient nearby the cued object during both inefficient and efficient visual search, in Experiment 2 we changed the invalid-off condition so that the nearby targets were now approximately $1 / 2$ the distance to the object than during Experiment 1 . This also created a spatial advantage for the invalid-off condition relative to the invalid-on condition.

Before moving on to Experiment 2, however, we will outline the findings of control Experiment S1, described in detail in the supplemental materials. In order to balance the side of the object (left/right) on which the invalid-off target was located in Experiment 1, we used two different display locations for the invalid-off condition. Each of these locations contained the target on $6.2 \%$ of trials, so that, together, the target probability in all invalid-off trials matched the probability in the invalid-on condition (12.4\%). One might argue that less attentional priority was allocated to the two invalid-off locations (vs. the single invalid-on location) solely on the basis of the probability with which targets would appear at those locations in Experiment 1. This probability difference could, thus, explain why the invalid-off RTs were so much slower than the invalid-on RTs. In Experiment S1, we replicated Experiment 1, but used only one invalid-off location: to the left of the object. The results (see the supplemental materials) indicated that the object-based effects were not different in Experiments 1 and S1, providing evidence that the target probability differences between invalid-on and invalid-off conditions in Experiment 1 do not explain the observed effects, and that target probability can be ruled out as a confound in this experiment (and subsequent ones).

\section{Experiment 2}

Our goal in this experiment was to test the limits of the attentional advantage afforded items on, and nearby, a selected object while observers engaged in a visual search. We moved the location of the invalid-off targets so that they were now spatially closer to the cue (see Fig. 1B) than in the invalid-on condition. This manipulation allowed us to measure the spatial gradient surrounding the cued object during both efficient and inefficient visual search conditions, the primary goal of the present work. The double-rectangle paradigm is predicated on the fact that both on-object and off-object locations are equidistant from the cue, suggesting that performance to targets at these locations should be comparable if only spacebased selection was occuring, whereas on-object performance should be advantaged if object-based selection was occuring. However, if the off-object targets are placed closer to the spatial cue (as we did in Exp. 2), we should see a reduction in the magnitude (or even elimination) of the OBA advantage.

\section{Method}

Participants A group of 32 naïve participants (mean age = 30.0; 19 males, 13 females) were recruited from the Pittsburgh university community, as in Experiment 1.

Apparatus, stimuli, design, and procedure All aspects of the experiment were identical to those of Experiment 1, with the following exception: Targets in the invalid-off condition were moved horizontally closer to the rectangle. This caused the distance between the cue and target to be $6.7^{\circ}$ in the invalid-off condition, while remaining $10.1^{\circ}$ in the invalidon condition. The same number of trials, conditions, randomization, and target probabilities were used as in Experiment 1. Half of the participants were assigned a target color of green, and the other half orange. 


\section{Results and discussion}

The data, displayed in Fig. 3, showed increases in RTs between target location conditions that were slightly different for the two distracter color conditions. Homogeneous distracters produced the following ordering of target location RTs: valid $<$ invalid-on $=$ invalid-off $<$ background. Heterogeneous distracters produced the following ordering of target location RTs: valid $<$ invalid-on $<$ invalid-off $<$ background. Number of distracters did not increase RTs in the homogeneous condition, but it did in the heterogeneous condition, though again only for the background target location trials.

Anticipatory responses (less than 150-ms RTs; $M=2.1$ trials/participant, $S D=2.9$ ) and incorrect trials were excluded from the analyses. As in Experiment 1, accuracy was at or near ceiling in all conditions (overall accuracy: $M=.971, S D=$ .025). An independent-samples $t$ test indicated no accuracy difference between green-target $(M=.978, S D=.013)$ and

\section{A}

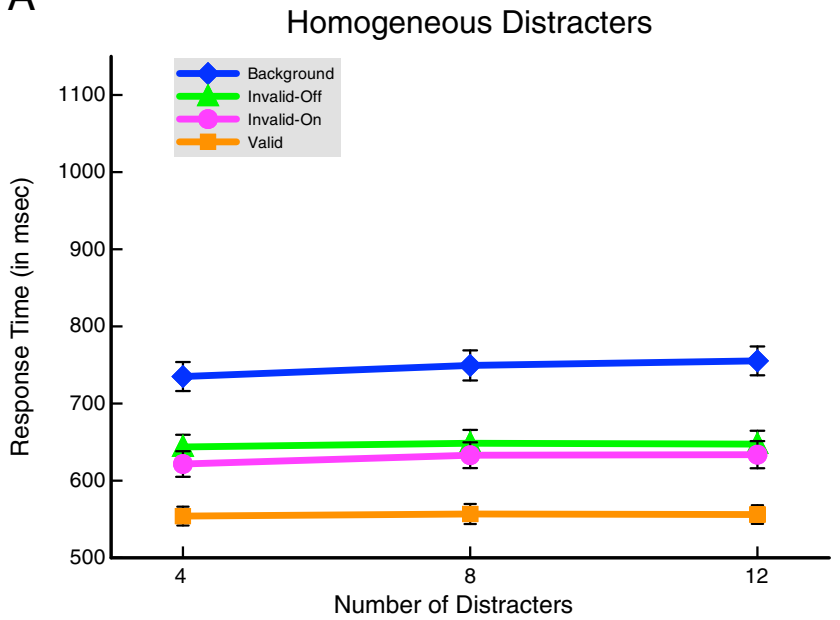

B

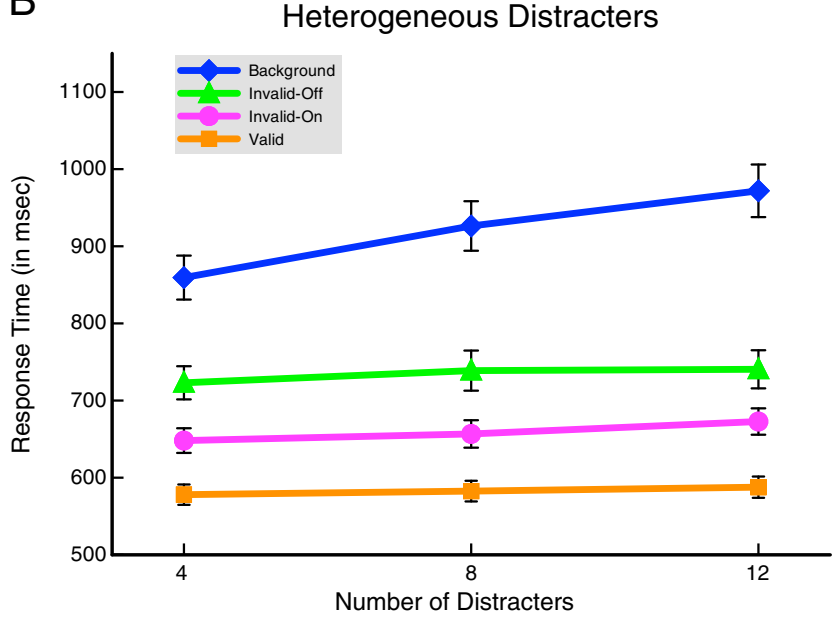

Fig. 3 Response time data for Experiment 2. The four target location condition RTs are plotted against the number of distracters for the homogeneous (A) and heterogeneous (B) distracter conditions orange-target $(M=.963, S D=.031)$ participants, $t(30)=$ $1.800, p=.082, d=0.66$. Accuracy was also not significantly different on homogeneous distracter trials $(M=.971, S D=$ $.026)$ and on heterogeneous distracter trials $(M=.970, S D=$ $.025)$, paired $t(31)=0.396, p=.695, d=0.04$. Other planned tests on accuracy were not significant.

An omnibus ANOVA on RTs yielded no significant main effect or interactions with the between-subjects factor Target Color. Therefore, all further analyses are collapsed across this manipulation. A within-subjects three-way ANOVA (see Fig. 3) with the factors Distracter Colors (homogeneous, heterogeneous), Target Location (valid, invalid-on, invalid-off, background), and Number of Distracters (four, eight, 12) revealed significant main effects of distracter colors $[F(1,31)=$ $\left.37.03, M S E=32,592.9, p<.001, \eta_{\mathrm{p}}{ }^{2}=.54\right]$, target location $\left[F(3,93)=186.16, M S E=12,705.0, p<.001, \eta_{\mathrm{p}}{ }^{2}=.86\right]$, and number of distracters $[F(2,62)=66.03, M S E=637.1, p<$ $\left..001, \eta_{\mathrm{p}}{ }^{2}=.68\right]$. This was qualified by significant interactions between distracter colors and target location $[F(3,93)=63.98$, $\left.M S E=3,488.5, p<.001, \eta_{\mathrm{p}}{ }^{2}=.67\right]$, distracter colors and number of distracters $[F(2,62)=28.97, M S E=552.1, p<$ $\left..001, \eta_{\mathrm{p}}{ }^{2}=.48\right]$, target location and number of distracters $[F(6$, $\left.186)=26.82, M S E=472.2, p<.001, \eta_{\mathrm{p}}{ }^{2}=.46\right]$, and a significant three-way interaction $[F(6,186)=13.29, M S E=$ 513.6, $\left.p<.001, \eta_{\mathrm{p}}{ }^{2}=.30\right]$.

In order to confirm that our distracter colors manipulation successfully encouraged efficient versus inefficient visual search, as in Experiment 1, post-hoc analyses using Tukey's HSD indicated that the RTs to background targets increased significantly with additional heterogeneous (all $p \mathrm{~s}<.01$; $14.07 \mathrm{~ms} /$ item), but not with additional homogeneous (all $\mathrm{ps}$ $>.05 ; 2.53 \mathrm{~ms} /$ item) distracters.

Having established the efficient versus inefficient search mode distinction, we then measured the effects of space- and object-based selection under the two search modes. As in Experiment 1, the space-based effects (invalid-on - valid) were equivalent during inefficient and efficient search (Tukey's HSD $p>.05$; see Table 1), providing further evidence that targets appearing on the object are shielded against the effects of search efficiency. Also, post-hoc Tukey tests revealed significant object-based effects (invalid-off - invalid-on) during both the heterogeneous and homogeneous distracter conditions (all $p \mathrm{~s}<.05$ ). Additionally, moving the invalid-off targets closer to the exogenous cue reduced the RT for these nearby targets. However, during both distracter conditions, participants were not faster in the invalid-off than in the invalid-on condition, despite invalid-on targets being located $\sim 3.4^{\circ}$ farther from the cue.

Of additional interest is the difference in the object-based effects between inefficient and efficient search conditions. Although, as in Experiment 1, the object-based effects were significant for both inefficient and efficient search, here the magnitude of this effect was greatly diminished, relative to the first 
experiment (see Table 1) by virtue of the invalid-off targets appearing nearer to the object in Experiment 2. However, further examination of the object-based effects revealed that the difference in mean invalid-off RTs for inefficient versus efficient search (see Table 2) was not statistically different between Experiments 1 and 2 [independent-samples $t(62)=$ $1.219, p=.228, d=0.31]$. This result suggests that distance from the object does not interact with search mode, and that OBA mechanisms may be independent from those involved in visual search (see the General Discussion).

In Experiment 3, we moved the locations of the invalid-off object condition yet again, so that targets were now approximately $1 / 2$ the distance to the object, as compared with Experiment 2, and $1 / 4$ the distance, as compared with Experiment 1 (see Fig. 1C). This would create a substantial spatial advantage for the invalid-off condition relative to the invalid-on condition and allow us to gain a more complete picture of the spatial gradient around the object during both efficient and inefficient search.

\section{Experiment 3}

Our goal in this experiment was to test the extreme limits of the OBA advantage while participants engaged in a visual search. We, again, moved the location of the targets during the invalid-off condition so that they were now only $5.3^{\circ}$ from the cue; invalid-on targets were still $10.1^{\circ}$ away from the cue.

\section{Method}

Participants Sixteen naïve participants (mean age $=25.6$; eight male, eight female) recruited from the Pittsburgh university community consented to participate for pay.

Apparatus, stimuli, design, and procedure All aspects of the experiment were identical to those of Experiment 2, with

Table 2 Invalid-off condition overall mean response times (in milliseconds) for all experiments, along with differences between inefficient and efficient search (in milliseconds)

\begin{tabular}{llll}
\hline & $\begin{array}{l}\text { Inefficient } \\
\text { Search }\end{array}$ & $\begin{array}{l}\text { Efficient } \\
\text { Search }\end{array}$ & $\begin{array}{l}\text { Inefficient - Efficient } \\
\text { Difference }\end{array}$ \\
\hline Exp. 1 & $807(29)$ & $693(19)$ & $114(16)^{*}$ \\
Exp. 2 & $734(24)$ & $647(17)$ & $87(16)^{*}$ \\
Exp. 3 & $728(42)$ & $634(27)$ & $94(27)^{*}$ \\
Exp. 4 (near) & $695(31)$ & $613(26)$ & $83(19)^{*}$ \\
Exp. 4 (far) & $804(39)$ & $667(27)$ & $137(22)^{*}$ \\
Exp. S1 & $794(29)$ & $652(19)$ & $142(16)^{*}$ \\
\hline
\end{tabular}

Values in parentheses are SEMs. Asterisks indicate significant differences $($ all $p \mathrm{~s}<.01)$ the following exception: Targets in the invalid-off condition were moved horizontally closer to the rectangle. This caused the distance between the cue and target to be $5.3^{\circ}$ in the invalid-off condition, while remaining $10.1^{\circ}$ in the invalidon condition (see Fig. 1C). Note that the invalid-off target letters were only $0.75^{\circ}$ from the near horizontal border of the rectangle, and (given the $0.5^{\circ}$ letter width) were, therefore, positioned essentially as close as possible to the rectangle border. The same number of trials, conditions, randomization, and target probabilities were used as in Experiment 1. In addition, because target color had shown no effects in the data from the previous two experiments, we assigned all participants a target color of green and tested half the number of participants as in either of the first two experiments.

\section{Results and discussion}

The data, displayed in Fig. 4, showed increases in RTs between the target location conditions, in the following order: valid $<$ invalid-on $=$ invalid-off $<$ background. This held for both homogeneous and heterogeneous distracters. Number of distracters did not increase RTs in the homogeneous condition, but it did in the heterogeneous condition, though again only for the background target location trials.

As previously, anticipatory responses (less than 150-ms RTs; $M=1.8$ trials/participant, $S D=3.4$ ) and incorrect trials were excluded from the analyses. Participant accuracy was, once again, at or near ceiling in all conditions (overall accuracy $M=.953, S D=.034)$. Accuracy did not differ between homogeneous $(M=.951, S D=.040)$ and heterogeneous $(M=$ $.956, S D=.030)$ distracter trials, paired $t(15)=1.081, p=$ $.297, d=0.16$.

A within-subjects three-way ANOVA on RTs (see Fig. 4) with the factors Distracter Colors (homogeneous, heterogeneous), Target Location (valid, invalid-on, invalid-off, background), and Number of Distracters (four, eight, 12) revealed significant main effects of distracter colors $[F(1,15)=21.87$, $\left.M S E=43,770.0, p<.001, \eta_{\mathrm{p}}{ }^{2}=.59\right]$, target location $[F(3,45)=$ 69.38, MSE $\left.=19,567.8, p<.001, \eta_{\mathrm{p}}{ }^{2}=.82\right]$, and number of distracters $\left[F(2,30)=18.14, M S E=1,325.8, p<.001, \eta_{\mathrm{p}}{ }^{2}=\right.$ .55]. This was qualified by significant interactions between distracter colors and target location $[F(3,45)=25.05, M S E=$ 4,124.7, $\left.p<.001, \eta_{\mathrm{p}}{ }^{2}=.63\right]$, distracter colors and number of distracters $\left[F(2,30)=6.24, M S E=1,425.2, p<.001, \eta_{\mathrm{p}}{ }^{2}=.29\right]$, target location and number of distracters $[F(6,90)=10.75, M S E$ $\left.=1178.3, p<.001, \eta_{\mathrm{p}}{ }^{2}=.42\right]$, and a significant three-way interaction $\left[F(6,90)=4.61, M S E=691.6, p<.001, \eta_{\mathrm{p}}{ }^{2}=.24\right]$.

Post-hoc analyses using Tukey's HSD, once again, confirmed that our distracter colors manipulation successfully encouraged inefficient versus efficient visual search: RTs to background targets increased significantly with additional heterogeneous (all $p \mathrm{~s}<.01 ; 16.1 \mathrm{~ms} / \mathrm{item})$, but not homogeneous (all $p \mathrm{~s}>.05 ; 5 \mathrm{~ms} /$ item), distracters. 


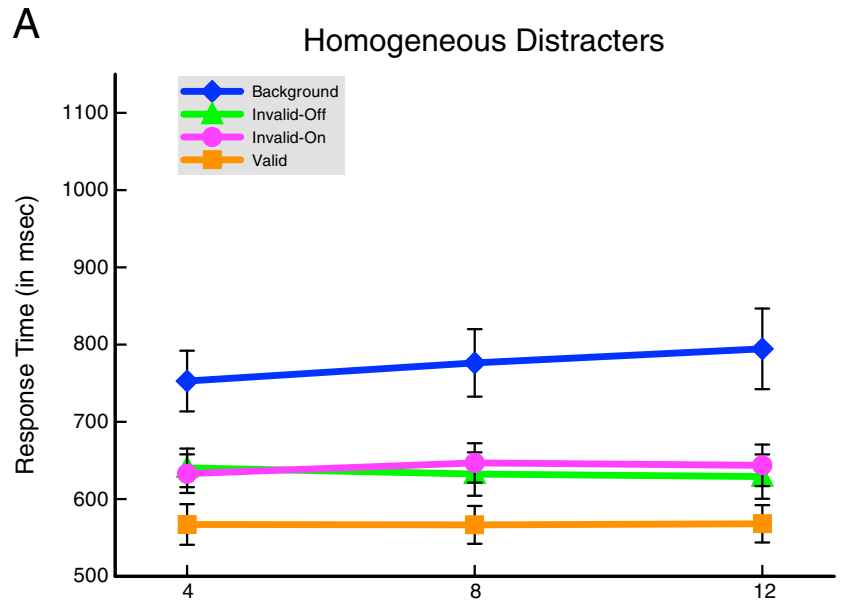

B

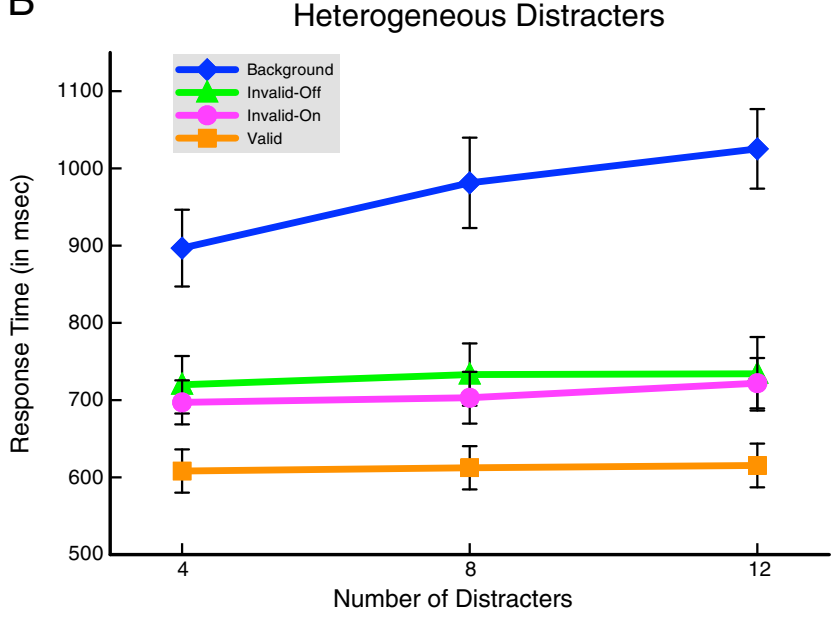

Fig. 4 Response time data for Experiment 3. The four target location condition RTs are plotted against the number of distracters for the homogeneous (A) and heterogeneous (B) distracter conditions

The space-based RT effects did not differ between inefficient $(M=90 \mathrm{~ms}, S D=87)$ and efficient $(M=74 \mathrm{~ms}, S D=66)$ search conditions [paired $t(15)=1.791, p=.10, d=0.29$ ], as previously. There were no significant RT differences between invalid-on and invalid-off trials during either efficient or inefficient search (all Tukey test $p \mathrm{~s}>.05$ ), and these conditions were not different from one another [paired $t(15)=1.631, p=$ $.12, d=0.51]$. Therefore, despite the large spatial advantage provided to invalid-off targets, RTs in this condition were no faster than those to invalid-on targets, replicating and extending Kravitz and Behrmann's (2008) results to conditions in which the off-object target is both closer to the object's center of mass and closer to the spatial cue.

To further explore the elimination of object-based effects in this experiment (see Table 1) we focused, as previously, on the invalid-off condition RTs. Despite the eradication of objectbased effects, Table 2 shows that the invalid-off RT difference between inefficient and efficient search was once again significant [paired $t(15)=3.588, p=.003, d=0.69$ ]. Furthermore, a one-way ANOVA confirmed that this difference did not change across the three experiments conducted $[F(2,77)=$ $\left.0.72, M S E=8,566.3, p=.488, \eta_{\mathrm{p}}{ }^{2}=.02\right]$.

These data provide insight into the attentional priority gradient surrounding a selected object. Kravitz and Behrmann (2008) previously reported that object-based attentional selection facilitates not only targets that appear within the bounds of the object, but also targets exterior to the object. They observed a gradient of attentional priority outside the attended object, whereby facilitation falls off linearly as a function of distance from the center of mass of the object. In those experiments, the authors probed locations both on and off the object, varying the off-object distance to the object's center of mass, which enabled them to map the attention gradient external to the object. However, the authors never tested a case in which the on-object target was farther from the object's center of mass than the off-object target. Therefore, it remained an open question whether off-object locations would yield faster RTs than on-object locations when the off-object location was closer to the center of mass. Through the present experiments, we are able to answer this question with a definitive "no." It seems that off-object locations are never able to produce faster RTs than on-object targets, even when (as in Exp. 3) the offobject target is directly adjacent to the attended object's center of mass. This further specification of the relationship between objectbased and space-based attentional selection in regions surrounding an attended object supports the notion that spatial and object representations interact in assigning scene-wide attentional priority.

\section{Experiment 4}

Our goal in this experiment was to test the limits of the OBA advantage while participants engaged in visual search in the context of a within-subjects manipulation. In the previous three experiments, we tesed a different group of individuals at each cue-target distance of the invalid-off object condition. It is possible that blocking this critical parameter could encourage participants to adopt a unique strategy in each experiment that might systematically affect results. Furthermore, manipulating an independent variable within subjects allows for a more powerful examination of the resulting effects than does the identical between-subjects manipulation.

Here, we tested the distance values used in Experiments 1 and 3 ; the invalid-near condition targets were $5.3^{\circ}$ from the cue, whereas invalid-far targets were $10.1^{\circ}$ away from the cue. All other parameters were identical to those in the previous three experiments.

\section{Method}

Participants Eighteen naïve participants (mean age $=27.2$; ten male, eight female) recruited from the Pittsburgh university community consented to participate for pay. 
Apparatus, stimuli, design, and procedure All aspects of the experiment were identical to those of Experiment 3, with the following exception: The targets in the invalid-off condition were at two possible distances from the rectangle. This caused the distance between the cue and target to be either $5.3^{\circ}$, in the invalid-near condition, or $10.1^{\circ}$, in the invalid-far condition (see Fig. 1C). The same randomization and number of trials were used as in Experiment 1. The following four trial types were defined by the target location: valid (64.8\%), invalid-on (12.4\%), invalid-near (6.2\%), invalid-far (6.2\%), and background (10.4\%) trials. In addition, because target color had shown no effects in the data from Experiments 1 and 2, we assigned all participants a target color of green and tested approximately half the number of participants as in either of the first two experiments.

\section{Results and discussion}

The data, displayed in Fig. 5, showed increases in RTs between target location conditions that were different for the two distracter color conditions. Homogeneous distracters produced the following ordering of target location RTs: valid $<$ invalid-on $=$ invalid-near $<$ invalid-far $<$ background. Heterogeneous distracters produced the following ordering of target location RTs: valid $<$ invalid-on $<$ invalid-near $<$ invalid-far $<$ background. Number of distracters did not increase RTs in the homogeneous condition, but it did in the heterogeneous condition, though again only for the background target location trials.

As previously, anticipatory responses (less than 150-ms RTs; $M=2.7$ trials/participant, $S D=4.3$ ) and incorrect trials were excluded from the analyses. Participants' accuracy was, once again, at or near ceiling in all conditions (overall accuracy $M=.965, S D=.036)$. Accuracy did not differ on homogeneous $(M=.978, S D=.192)$ and heterogeneous $(M=.959$, $S D=.443)$ distracter trials, paired $t(17)=2.011, p=.060, d=$ 0.56 .

A within-subjects three-way ANOVA on RTs (see Fig. 5) with the factors Distracter Colors (homogeneous, heterogeneous), Target Location (valid, invalid-on, invalid-near, invalid-far, background), and Number of Distracters (four, eight, 12) revealed significant main effects of distracter colors $[F(1$, $\left.17)=29.36, M S E=43,051.7, p<.001, \eta_{\mathrm{p}}{ }^{2}=.63\right]$, target location $\left[F(4,68)=125.39, M S E=8,091.9, p<.001, \eta_{\mathrm{p}}{ }^{2}=\right.$ $.88]$, and number of distracters $[F(2,34)=10.05, M S E=1$, $\left.303.5, p<.001, \eta_{\mathrm{p}}{ }^{2}=.37\right]$. These were qualified by significant interactions between distracter colors and target location $[F(4$, $\left.68)=41.08, M S E=2,944.4, p<.001, \eta_{\mathrm{p}}{ }^{2}=.71\right]$, distracter colors and number of distracters $[F(2,34)=5.40, M S E=$ $\left.1080.1, p<.01, \eta_{\mathrm{p}}{ }^{2}=.24\right]$, target location and number of distracters $\left[F(8,136)=7.63, M S E=958.7, p<.001, \eta_{\mathrm{p}}{ }^{2}=\right.$ $.31]$, and a significant three-way interaction $[F(8,136)=3.06$, $\left.M S E=840.8, p<.005, \eta_{\mathrm{p}}{ }^{2}=.15\right]$.
A

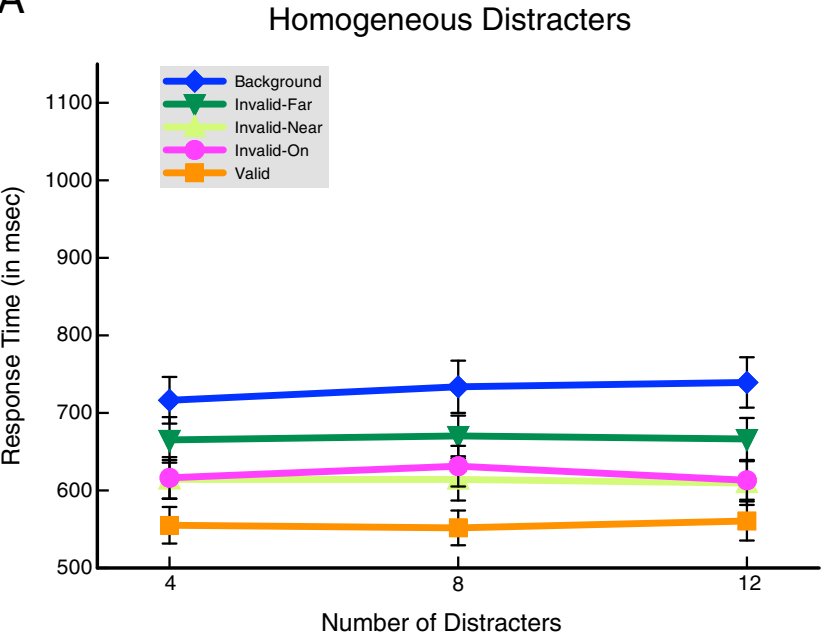

B

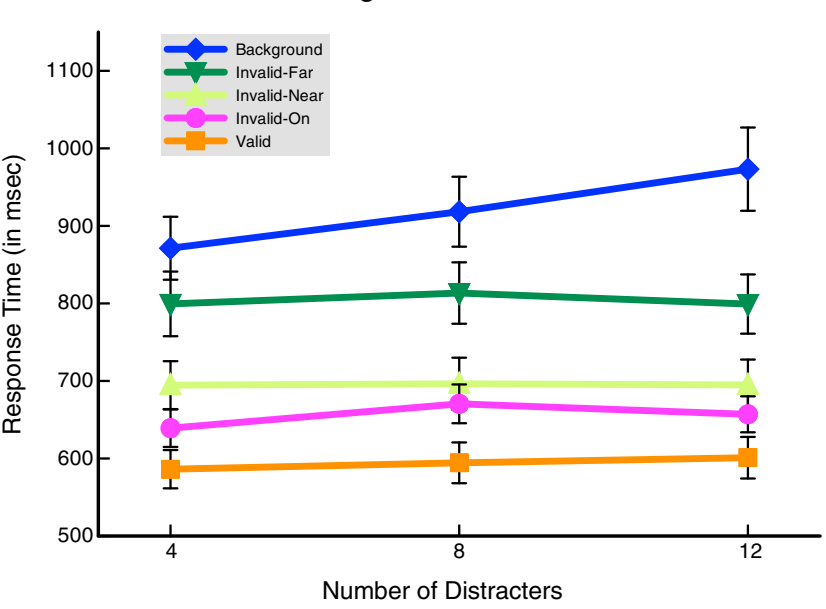

Fig. 5 Response time data for Experiment 4. The five target location condition RTs are plotted against the number of distracters for the homogeneous (A) and heterogeneous (B) distracter conditions

Post-hoc analyses using Tukey's HSD, once again, confirmed that our distracter colors manipulation successfully encouraged inefficient versus efficient visual search: RTs to background targets increased significantly with additional heterogeneous (all $p \mathrm{~s}<.01 ; 11 \mathrm{~ms} /$ item), but not homogeneous (all $p \mathrm{~s}>.01 ; 2.5 \mathrm{~ms} /$ item), distracters.

The space-based RT effects did not differ between inefficient $(M=62 \mathrm{~ms}, S D=51 \mathrm{~ms})$ and efficient $(M=64 \mathrm{~ms}, S D=$ $41 \mathrm{~ms}$ ) search conditions [paired $t(17)=0.295, p=.77, d=$ $0.04]$, as previously. There were no significant RT differences between invalid-on and invalid-near during efficient search (all Tukey test $p \mathrm{~s}>.05$ ). However, invalid-on and invalidnear were significantly different during inefficient search at each of the three (four, eight, and 12) distracter number conditions (all Tukey test $p \mathrm{~s}<.01$ ). All other conditions were significantly different from one another during both efficient and inefficient search (all Tukey test $p \mathrm{~s}<.01$ ). 
Therefore, as we previously showed in Experiment 3, despite the large spatial advantage provided to invalid-near targets, RTs in this condition were no faster than those to invalidon targets. However, as was previously shown in Experiment 1 , when targets were equidistant to the cue, the invalid-on RTs are faster than invalid-far RTs, presumably because they were located on the cued object.

To explore the modulation of object-based effects in this experiment (see Table 1), we focused on the invalid-near and invalid-far condition RTs. Despite the eradication of invalidnear object-based effects during efficient search [one-sample $t(17)=1.041, p=.312, d=0.50]$, Table 2 shows that the invalid-near RT difference between inefficient and efficient search was once again significant (Tukey test $p<.01$ ). This was obviously also the case for the invalid-far condition (Tukey test $p<.01$ ), in which significant object-based effects were observed during both efficient [one-sample $t(17)=$ $5.560, p<.001$ ] and inefficient [one-sample $t(17)=7.431, p$ $<.001]$ search. As is illustrated in Table 1, the mean objectbased effects for the invalid-far condition were more than three times larger during inefficient search $(M=149)$ than during efficient search $(M=47)$, similar to Experiment 1 (and Exp. S1).

To summarize, manipulating off-object target distance within subjects confirmed our findings from Experiments 13. Invalid-near targets produced object-based effects similar to those seen in Experiment $3,{ }^{2}$ and invalid-far targets produced object-based effects similar to those seen in Experiment 1. Furthermore, the space-based effects (as in previous experiments) did not differ between inefficient and efficient search conditions. We, therefore, conclude that blocking the cue-target distance parameter in Experiments 1-3 did not systematically affect the results by encouraging participants to adopt a unique strategy. It seems that participants produce the same data when the cue-target distance is randomized within the experiment.

\section{General discussion}

In this study, we probed the representational basis of attentional selection under varying conditions of search efficiency.

\footnotetext{
2 The only exception was that the invalid-near targets of Experiment 4 produced significant object-based effects during inefficient search, whereas the invalid-off targets of Experiment 3 failed to produce significant object-based effects. Although this point is interesting, our primary focus was on the difference in object-based effects between near and far invalid-off targets during inefficient search. The magnitudes of this difference for the between-subject's data $(117 \mathrm{~ms})$ and the within-subject's data $(109 \mathrm{~ms})$ are nearly identical, providing evidence that the betweensubjects and within-subjects manipulations were likely inherently the same. That is to say, participants were unlikely to be using any unique or unusual strategies as a result of distance being blocked in Experiments $1-3$.
}

More specifically, we combined the standard visual search and cued-rectangle paradigms to explore the interaction between search efficiency and object-based attentional selection. At the outset, we outlined two questions that our method is uniquely qualified to address. Below, we discuss our results in light of each of these.

\section{Is the prioritization search affected by mode of visual search?}

As we discussed in the introduction, the attentional prioritization hypothesis proposed by Shomstein and Yantis (2002) posits a two-stage selection process during object-based attentional selection: an automatic spatial gradient centered on the spatial cue, and a strategic selection based on target location probability. The combined attentional priority of these two stages of object-based selection determines the order in which participants will search the display for the presence of a target. In the paradigm employed in Experiment 1, the highest combined priority location is the valid location, followed by the invalid-on object location, then the invalid-off object location(s), and finally the background locations. The ordering of participants' visual search was borne out in the mean RT data for Experiment 1, in which the valid condition was fastest, followed by invalid-on, then invalid-off, and finally background. This pattern held true for both distracter color conditions, homogeneous and heterogeneous, which we showed were successful in promoting efficient and inefficient visual search, respectively. Thus, on the basis of the data from Experiment 1 alone, we would conclude that visual search efficiency (or mode) does not affect the prioritization search postulated in the attentional prioritization hypothesis.

However, whereas the ordering of the search was unaffected by search mode, the magnitude of the OBA advantage was strongly modulated by search mode. Experiments 1, 2, and 4 each yielded significantly different magnitudes of the OBA advantage (invalid-off RT - invalid-on RT) for efficient and inefficient search. This effect encourages all the more confidence in light of the extraordinary consistency of the spacebased effects (valid RT - invalid-on RT) during these identical conditions in all experiments (see Table 1). Furthermore, the magnitude of the OBA advantage during inefficient search (particularly in Exps. 1 and 4) was more than three times what is typically seen in the Egly et al. (1994) style, doublerectangle experiment (see the Discussion following Exp. 1). The data across experiments showed that the enormous OBA advantage that we observed during inefficient search was led by a slowing of RTs during the invalid-off condition (rather than a speeding of RTs during the invalid-on condition). As we discussed earlier (see the Discussion following Exp. 1), the invalid-off object target loses its status as a color singleton when the distracters are heterogeneous (the inefficient search condition). This leads to increased confusability between the 
target and distracters. We concluded that this increased confusability causes an alteration of attentional priority, such that the invalid-off object location is prioritized lower than the invalid-on object location. However, the invalid-off object location is still prioritized higher than the background items, due to the greater probability with which the target appears at the invalid-off object location(s). We believe this targetdistracter confusability is instantiated as a change in strategy, therefore affecting only the strategic stage of the attentional prioritization account. In summary, search efficiency has an indirect effect on the OBA prioritization search due to increased target-distracter confusability during inefficient search.

\section{Does the prioritization search share a common search mechanism with standard visual search processes?}

Possibly the most surprising observation in this study was the stability of the space-based effect (invalid-on RT - valid RT) across all conditions tested. None of the experiments showed a reliable difference in these effects as a function of search mode; there were also no significant effects of number of distracters (search slopes), and no significant differences in space-based effects across Experiments 1-4, either. The natural conclusion is that the distribution of attentional priority on the cued object is completely unaffected by search efficiency. So, as we established in the previous section, participants always begin their search for the target at the valid location and then turn to the invalid-on object location. The question then becomes: What happens when participants must search outside the selected object? Again, as we established above, the invalid-off object location is searched third. But, a priori, we did not know how search efficiency would affect invalid-off RTs. If we observed search slopes increasing with set size during inefficient search (and flat search slopes during efficient search), we could reasonably conclude that the prioritization search shared the same mechanism as the standard visual search. However, if we observed flat search slopes during both search modes, this would point to distinct mechanisms for the prioritization search and the standard visual search. The data unambiguously support the latter proposal: In all experiments that we tested, the invalid-off search slopes were flat during both efficient and inefficient search, unlike the background search slopes, which always increased with set size during inefficient search. This suggests, to us, that the standard visual search mechanism is different from the prioritization search proposed by the attentional prioritization hypothesis.

\section{Implications for the attentional prioritization hypothesis}

An additional advantage of the paradigm that we used in this study is that we could produce a rough, first-pass model of the interactions between the two stages of selection proposed by the attentional prioritization hypothesis of OBA. In all experiments, the target probabilities were equivalent for the invalidoff and invalid-on conditions. Thus, the strategic selection process should be identical across all experiments (though, as we pointed out above, this process is affected by visual search mode). Therefore, any change in performance (for a given search mode) across experiments is likely due to the location of the invalid-off item within the spatial gradient invoked by the automatic spatial selection stage. The plots in Fig. 6 show the OBA advantage (RT difference between invalid-on and invalid-off) as a function of distance to the spatial cue (in degrees of visual angle), separately for inefficient and efficient search. Examination of the regression line in each plot provides a sense of the relationship between cuetarget distance and object-based effects under these

\section{A}

\section{Efficient Search}

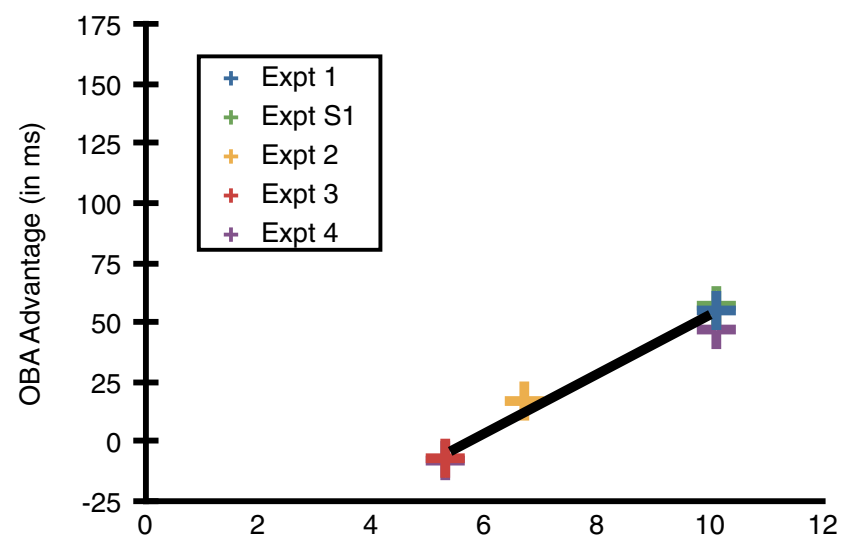

\section{B Inefficient Search}

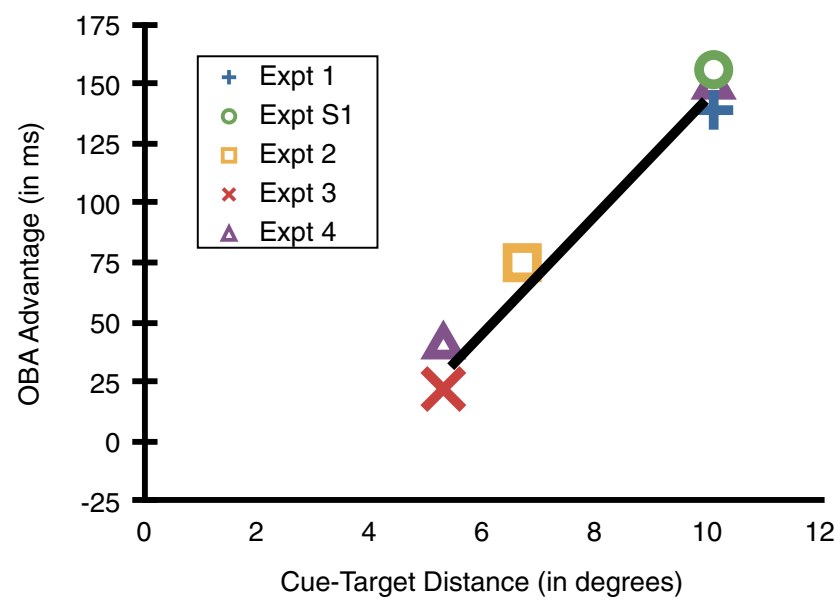

Fig. 6 Object-based attention (OBA) advantage as a function of distance to the spatial cue. Each data point is the mean effect across subjects taken from one of the five experiments reported in this study. As cue-target distance increases, the OBA advantage linearly increases, as well. However, efficient search (A) yields a less steep slope than does inefficient search (B) 
conditions. These data indicate that the spatial gradient is well explained by a linear fit (inefficient: $r=.989, m=24, b=-93$; efficient: $r=.990, m=12, b=-71)$.

As is indicated by the slopes $(m)$ of these regression lines, the OBA advantage grows faster (as a function of increased off-object target distance) during inefficient than during efficient search. Thus, when participants are engaged in an efficient visual search, as off-object targets are moved farther from the cue, the additional RT advantage for the on-object target is somewhat moderate. However, when participants are engaged in an inefficient visual search, as off-object targets are moved farther from the cue, the additional RT advantage for the on-object target is much more substantial. This suggests that the spatial gradient falls off more quickly (as one moves away from the spatial cue, where it is centered) during inefficient than during efficient search.

Furthermore, the $x$-intercepts of these regression lines yield the equivalence between cue-target distance and the spatial gradient. That is to say, during efficient search, a 5.9-deg advantage for the invalid-off object target over the invalid-on object target should provide equated RTs for these two target locations. However, during inefficient search, only a 3.9-deg advantage for the invalid-off object target is required to provide equated RTs. This analysis makes clear the trade-off between cue-target distance and object-based prioritization under varied search efficiency. When search is efficient, objectbased selection is more difficult to overcome, thus requiring a much stronger weighting from the spatial gradient (achieved by moving the off-object target closer to the cue) in order to equate performance at the two invalid locations. When search is inefficient, object-based selection is easier to overcome, requiring only a moderately stronger weighting from the spatial gradient in order to equate performance.

\section{Visual search models}

Although the focus of this study has been to shed light on models of object-based attentional selection, we acknowledge that the visual search literature provides a set of models that may also be consistent with many of the present findings. The Guided Search model (Wolfe, 1994), for example, allows (though, not explicitly) for a visual search in which the combined effects of spatial selection (via exogenous cue), target probability, feature template activation, and perceptual organization are all helping direct attentional priority. Other models (e.g., Duncan \& Humphreys, 1989; Treisman, 1982) also do not explicitly deny that this combination of factors may guide how a visual search like the one employed here might work. However, these models might have difficulty explaining the results from the double-rectangle cueing paradigm of Egly and colleagues (1994), in which a same-object advantage is generated without explicit differences in the target feature. Models of object-based attention have arisen to help us understand what happens when attention selects information on the basis of objects. These models (specifically, the prioritization account of Shomstein \& Yantis, 2002) have begun including a limited visual search in order to better explain recent findings. Visual search models, to our knowledge, have not been extended to conditions under which cued perceptual objects are present in the display (as we noted, however, these models don't explicitly prevent this). Therefore, it may be that visual search and object-based attention models are wholly consistent in such contexts (though this theoretical argument is outside the scope of the present article). Indeed, we believe that one of the contributions of this study is the marrying of these fields via the use of a novel paradigm that can be manipulated to explicate future combined models of visual search and object-based attention.

\section{Conclusions}

We have developed a new method for measuring the effects of object-based attention in the context of a larger-scale visual search, in order to test critical aspects of the attentional prioritization hypothesis of object-based selection. We observed that the mode of information transmission (search efficiency) affects the magnitude, but not the ordering, of the prioritization search. The data also suggest that standard spatial visual search and object-based prioritization search may rely on distinct mechanisms. Our data inform a course model of the attentional prioritization hypothesis that explicates the tradeoff between cue-target distance and object-based prioritization under conditions of varied search efficiency. These data are consistent with more general proposals that the influence of object representations on visual attention is under voluntary control (Drummond \& Shomstein, 2010; Greenberg, 2009; Greenberg \& Gmeindl, 2008). Finally, Shomstein (2012) recently challenged the field of object-based attention to move beyond simple on/off demonstrations, and instead to focus on modulatory behaviors that depend less on null results. Here, we provide such "stronger forms of inference" (Shomstein, 2012) by measuring the modulation of robust object-based effects during the manipulation of search efficiency.

Author note This research was supported by NIH Grant Nos. RO1MH54246 (to M.B.) and T32-MH19983 (to A.S.G.). We thank Kamy Wakim and Kayla Zamora for help with data collection.

\section{References}

Brainard, D. H. (1997). The Psychophysics Toolbox. Spatial Vision, 10, 433-436. doi:10.1163/156856897X00357

Chou, W. L., \& Yeh, S. L. (2012). Object-based attention occurs regardless of object awareness. Psychonomic Bulletin \& Review, 19, 225231. doi:10.3758/s13423-011-0207-5 
Drummond, L., \& Shomstein, S. (2010). Object-based attention: Shifting or uncertainty? Attention, Perception, \& Psychophysics, 72, 1743 1755. doi:10.3758/APP.72.7.1743

Duncan, J. (1984). Selective attention and the organization of visual information. Journal of Experimental Psychology: General, 113, 501517. doi:10.1037/0096-3445.113.4.501

Duncan, J., \& Humphreys, G. W. (1989). Visual search and stimulus similarity. Psychological Review, 96, 433-458. doi:10.1037/0033295X.96.3.433

Egeth, H., Jonides, J., \& Wall, S. (1972). Parallel processing of multielement displays. Cognitive Psychology, 3, 674-698.

Egly, R., Driver, J., \& Rafal, R. D. (1994). Shifting visual attention between objects and locations: Evidence from normal and parietal lesion subjects. Journal of Experimental Psychology: General, 123, 161-177. doi:10.1037/0096-3445.123.2.161

Goldsmith, M. (1998). What's in a location? Comparing object-based and space-based models of feature integration in visual search. Journal of Experimental Psychology: General, 127, 189-219. doi:10.1037/ 0096-3445.127.2.189

Greenberg, A. S. (2009). Uncertainty as a guiding principle in the strategic allocation of attention to objects [Ph.D. dissertation]. Baltimore, MD: Johns Hopkins University.

Greenberg, A. S., \& Gmeindl, L. (2008). Strategic control of attention to objects and locations. Journal of Neuroscience, 28, 564-565. doi: 10.1523/JNEUROSCI. 4386-07.2008

Hollingworth, A., Maxcey-Richard, A. M., \& Vecera, S. P. (2012). The spatial distribution of attention within and across objects. Journal of Experimental Psychology: Human Perception and Performance, 38, 135-151. doi:10.1037/a0024463

Kravitz, D. J., \& Behrmann, M. (2008). The space of an object: Object attention alters the spatial gradient in the surround. Journal of Experimental Psychology: Human Perception and Performance, 34, 298-309. doi:10.1037/0096-1523.34.2.298

Lin, S.-Y., \& Yeh, S.-L. (2011). First come, first served? Influence of changed object configuration on object-based attention. Attention, Perception, \& Psychophysics, 73, 678-687. doi:10.3758/s13414010-0078-2

Lin, S.-Y., \& Yeh, S.-L. (2012). Every moment counts: Smooth transitions of object boundaries reflect constant updating in object-based attention. Attention, Perception, \& Psychophysics, 74, 533-539. doi:10.3758/s13414-011-0245-0

Pelli, D. G. (1997). The VideoToolbox software for visual psychophysics: Transforming numbers into movies. Spatial Vision, 10, 437-442. doi:10.1163/156856897X00366

Pilz, K. S., Roggeveen, A. B., Creighton, S. E., Bennett, P. J., \& Sekuler, A. B. (2012). How prevalent is object-based attention? PLoS ONE, 7, e30693. doi:10.1371/journal.pone.0030693

Rensink, R. A., \& Enns, J. T. (1995). Pre-emption effects in visual search: Evidence for low-level grouping. Psychological Review, 102, 101130. doi:10.1037/0033-295X.102.1.101

Shomstein, S. (2012). Object-based attention: Strategy versus automaticity. WIRE's Cognitive Science, 3, 163-169.

Shomstein, S., \& Yantis, S. (2002). Object-based attention: Sensory modulation or priority setting? Perception \& Psychophysics, 64, 41-51. doi:10.3758/BF03194556

Treisman, A. (1982). Perceptual grouping and attention in visual search for features and for objects. Journal of Experimental Psychology: Human Perception and Performance, 8, 194-214. doi:10.1037/ 0096-1523.8.2.194

Treisman, A. M., \& Gelade, G. (1980). A feature-integration theory of attention. Cognitive Psychology, 12, 97-136. doi:10.1016/00100285(80)90005-5

Vecera, S. P., \& Farah, M. J. (1994). Does visual attention select objects or locations? Journal of Experimental Psychology: General, 123, 146 160. doi:10.1037/0096-3445.123.2.146

Wolfe, J. M. (1994). Guided Search 2.0: a revised model of visual search Psychonomic Bulletin \& Review, 1, 202-238. doi:10.3758/ BF03200774

Wolfe, J. (1998). Visual search. In H. Pashler (Ed.), Attention (pp. 13-73). Hove, UK: Psychology Press.

Wolfe, J., \& Horowitz, T. S. (2004). What attributes guide the deployment of visual attention and how do they do it? Nature Reviews Neuroscience, 5, 495-501. doi:10.1038/nrn1411

Yeari, M., \& Goldsmith, M. (2010). Is object-based attention mandatory? Strategic control over mode of attention. Journal of Experimental Psychology: Human Perception and Performance, 36, 565-579. 